\title{
Real Life Treatment of Hepatocellular Carcinoma: Impact of Deviation from Guidelines for Recommended Therapy
}

\author{
Alzhraa Alkhatib ${ }^{1}$, Asmaa Gomaa ${ }^{2}$, Naglaa Allam²*, Eman Rewisha², Imam \\ Waked $^{2}$
}

\begin{abstract}
Background: Real life management of hepatocellular carcinoma occasionally deviates from guidelines for recommended therapy. Aims: To evaluate how frequent this deviation happens in our center and assess its impact on outcome. Materials and Methods: The treatment of 770 patients (87\% males, mean age 57.8 years) was analyzed and the effect of deviation on outcome over 36 months was examined. Results: Of Barcelona Clinic liver cancer stages 0 and A patients, $65.8 \%$ received resection, ablation, liver transplantation or transarterial chemoembolisation for unresectable tumors more than $5 \mathrm{~cm}$ in diameter, and $34.2 \%$ received treatment recommended for later stages. Of stage $B$ patients, $62.2 \%$ received recommended therapy, $34.3 \%$ of patients received supportive therapy or sorafenib and $3.5 \%$ received upward treatment stage migration. Among stage $C$ patients, $\mathbf{7 . 6 \%}$ received sorafenib, and most $\mathbf{( 7 9 . 2 \% )}$ ) were given supportive care. Deviation from recommended therapy occurred in $34.2 \%, 37.7 \%$, and $92.4 \%$ in stages 0 -A, B and C. Survival of stage 0 -A patients who received downwards treatment stage migration was lower than those who received recommended treatment $(p<0.001)$. Upward treatment stage migration in stages B,C and D did not improve survival compared to those who received recommended treatment. Conclusions: Deviation from recommended therapy had a negative impact on survival in Barcelona Clinic liver cancer stage A patients.
\end{abstract}

Keywords: AASLD guidelines - barcelona clinic liver cancer - hepatocellular carcinoma - survival

Asian Pac J Cancer Prev, 16 (16), 6929-6934

\section{Introduction}

Primary liver cancer is one of the most common cancers at the global level, accounting for half of all cancers in some developing countries (Su et al., 2013). The outcome of hepatocellular carcinoma (HCC) is related to the extent of both tumor and liver disease, which influence the applicability and efficacy of treatments. The BCLC staging system links disease stage with treatment modalities and with an estimation of life expectancy (Bruix and Sherman, 2011). Most treatment guidelines recommend therapy based on patient's BCLC stage (Bruix et al., 2001; Ryder, 2003; Parikh et al., 2008; Benson et al., 2009; Omata et al., 2010; Bruix and Sherman, 2011; EASL 2012). For patients at stage 0, radical therapies can completely eradicate the tumor. Stage A patients should be evaluated for surgical resection or local ablations. Transarterial chemoembolization (TACE) is the preferred option for stage B patients. Sorafenib is the sole treatment that has shown a positive impact on survival for patients with vascular invasion, extrahepatic spread and/or constitutional symptoms in stage C. Stage D patients should receive only symptomatic care, as their expected survival is less than three months (Llovet et al., 2008;
Bruix and Sherman, 2011; Lope et al., 2012).

Non-adherence to international guidelines is common (Borzio and Saco, 2013), even in resource-rich settings. How often real-life management deviates from recommended therapy in a resource-limited setting, and the impact of deviation on patients' survival has not been evaluated and is the subject of this analysis.

\section{Materials and Methods}

This study was conducted prospectively on 770 Egyptian patients with HCC managed in a tertiary referral center in 2011 and 2012. The study conformed to the ethical guidelines of Declaration of Helsinki, and was approved by the Institutional Review Board. Written informed consent was obtained from each patient. The diagnosis of HCC was mainly non-histological according to the American Association for the Study of Liver Disease (AASLD) criteria of 2005 (Bruix and Sherman, 2005).

Demographic information, etiology of liver disease, biochemical data including serum bilirubin, serum albumin, prothrombin time and concentration, ALT, AST, complete blood picture and serum alpha-fetoprotein (AFP) were evaluated for all patients. Presence of underlying 
cirrhosis, ascites and encephalopathy were assessed. Performance status was assessed as recommended by the ECOG (Eastern Cooperative Oncology Group) (Oken et al., 1982). Assessment of hepatic function based on Child-Turcotte-Pugh (CTP) classification was recorded. Hepatocellular carcinoma characteristics including number of nodules, maximal diameter of the largest nodule, extrahepatic spreading and vascular invasion were detected by dynamic contrast-enhanced CT or MRI. Patients were staged according to the revised BCLC classification of 2009 (Forner et al., 2010).

A multidisciplinary team (a hepatologist, oncologist, surgeon, transplant surgeon, radiologist and invasive radiologist) made therapeutic decisions for each patient according to the treatment algorithm of therapy recommended by the AASLD for the different stages of the BCLC. Liver transplantation (LTx) was recommended for patients with single tumor less than $5 \mathrm{~cm}$ or $\leq 3$ nodules $\leq 3 \mathrm{~cm}$ (within Milan criteria) and AFP level <1,000ng/ $\mathrm{ml}$. Hepatic resection was recommended for patients with single tumor with well-preserved liver function (CTP A) without evidence of portal hypertension. Radiofrequency ablation (RFA) was recommended for patients with up to three lesions up to $5 \mathrm{~cm}$ and with compensated liver disease (CTP A or B). Ethanol injection was done in tumors $<2 \mathrm{~cm}$ and technically not feasible for RFA. For larger tumors without vascular invasion or extra-hepatic spread and with compensated liver cirrhosis (CTP A or B) TACE was the recommended treatment. Sorafenib was recommended for patients with large multinodular disease not suitable for TACE or patients with vascular invasion, who were within CTPA or B. Patients with decompensated liver disease (CTP C) or with Performance status (PS) $>2$ received only supportive care.

The actual treatment received by the patients was determined, and it was noted whether it deviated from the recommended therapy or not. The reason for the deviation was noted. Deviations from the AASLD recommended therapy for the BCLC stage was categorized as "upward treatment stage migration"; a group that received treatment which according to the AASLD recommendation was indicated for the previous BCLC stage, or "downward treatment stage migration"; those who received treatment which according to AASLD recommendation was indicated for the later BCLC stage. The patients were followed and the survival was compared in those treated according to BCLC recommendation and those treated differently.

Table 1. Demographic data, Tumour Characteristics \&the Received Treatment in the studied Patients

\begin{tabular}{|c|c|}
\hline Variable & Value \\
\hline Age (years), m (range) & $57.8(30-85)$ \\
\hline Male /Female, n (\%) & $669(86.9) / 101(13.1)$ \\
\hline \multicolumn{2}{|l|}{ Etiology of liver disease, $\mathrm{n}(\%)$} \\
\hline Hepatitis C/B/ Combined C \&B/ Non C or B & $723(93.9) / 17(2.2) / 2(0.3) / 28(3.6)$ \\
\hline Alpha-fetoprotein $(\mathrm{ng} / \mathrm{ml})$, mean (range) & $9225(0.5-2395406)$ \\
\hline Performance status $0 / 1 / 2 / 3, \%$ & $69.1 / 21.7 / 8.3 / 0.9$ \\
\hline \multicolumn{2}{|l|}{ Child-Pugh class, n (\%) } \\
\hline $\mathrm{A} / \mathrm{B} / \mathrm{C}$ & $408(53) / 292(37.9) / 70(9.1)$ \\
\hline Tumor size $(\mathrm{cm})$, mean (range) & $5.7(1-27)$ \\
\hline \multicolumn{2}{|l|}{ Number of focal lesions, $\mathrm{n}(\%)$} \\
\hline One /Two/Three/ More than three & $454(59) / 77(10) / 12(1.5) / 227(29.5)$ \\
\hline \multicolumn{2}{|l|}{ Location of tumor, $\mathrm{n}(\%)$} \\
\hline Right lobe / Left lobe/ Both lobes & $487(63.3) / 112(14.5) / 171(22.2 \%)$ \\
\hline Presence of portal vein thrombosis, $\mathrm{n}(\%)$ & $165(21.4)$ \\
\hline Metastasis, n (\%) & $80(10.4)$ \\
\hline Ascites, n (\%) & 253(32.9) \\
\hline \multicolumn{2}{|l|}{ Received treatment in BCLC stage $0+A(n=292), n(\%)$} \\
\hline Resection/PEI/RFA/TACE/MWA/BSC/Sorafenib/LT & $19(6.5) / 5(1.7) / 64(21.9) / 132(45.2) / 4(1.4) / 51(17.5) / 3(1) / 14(4.8)$ \\
\hline \multicolumn{2}{|l|}{ Received treatment in BCLC stage B $(\mathrm{n}=143), \mathrm{n}(\%)$} \\
\hline RFA/TACE/BSC/Sorafenib/LT & $5(3.5) / 86(60.1) / 34(23.8) / 15(10.5) / 3(2.1)$ \\
\hline \multicolumn{2}{|l|}{ Received treatment in BCLC stage C $(\mathrm{n}=265), \mathrm{n}(\%)$} \\
\hline $\begin{array}{l}\text { Resection/RFA/TACE/RFA+TACE/BSC/Sorafenib/LT/ } \\
\text { Chemo/Radio }\end{array}$ & $\begin{array}{c}2(0.8) / 3(1.1) / 22(8.3) / 1(0.4) / 210(79.2) / 20(7.5) / 3(1.1) / 3(1.1) / \\
1(0.4)\end{array}$ \\
\hline \multicolumn{2}{|l|}{ Received treatment in BCLC stage $\mathrm{D}(\mathrm{n}=70), \mathrm{n}(\%)$} \\
\hline TACE/BSC/LT & $1(1.4) / 63(90) / 6(8.6)$ \\
\hline
\end{tabular}

Demographic data, Tumour characteristics \& the received Treatment in the studied patients 


\section{Statistical analysis}

Parametric data was presented as mean and standard deviation, while non-parametric data was presented as median and percentiles for quantitative variables. Qualitative data was summarized by frequencies. The differences between categorical variables were analyzed with a Chi-square test. Length of survival was calculated from the date of HCC diagnosis to the date of death or, in the case of survivors, the date of the last follow up visit. Univariate analysis for survival was performed using the Kaplan-Meier method with the log rank test to verify significance of differences (Kaplan and Meier, 1958). Survival was plotted as Kaplan-Meier graphs. A p value $<0.05$ was considered statistically significant.

\section{Results}

The baseline characteristics and the treatment of the studied patients are shown in Table 1 . The mean age was 57.8 years (range 30-85 years) and male gender

Table 2. Reasons for divergence from recommendations of American Association for the Study of Liver Disease

\begin{tabular}{lrl}
\hline $\begin{array}{l}\text { BCLC } \\
\text { stage }\end{array}$ & $\begin{array}{c}\text { Number of } \\
\text { deviation }\end{array}$ & \multicolumn{1}{c}{$\begin{array}{c}\text { Causes of } \\
\text { deviations }\end{array}$} \\
\hline 0+A & 16 & Infiltrative lesion \\
& 3 & Exophytic in the presence \\
& & of Portal hypertension \\
& 6 & Old age \\
& 1 & Co-morbidities \\
& 14 & Patient willing \\
& 28 & Low accessibility \\
& 32 & Portal hypertension \& inaccessible \\
B & 32 & site for local ablation \\
& 8 & Infiltrative lesion \\
& 1 & Old age \\
C & 13 & Patient willing \\
& 89 & Impaired liver function \\
& 18 & Old age \\
& 5 & Co-morbidities \\
& 35 & Patient willing \\
& 95 & Low accessibility \\
& 1 & Patient willing \\
\hline
\end{tabular}

predominated $(86.9 \%)$. Chronic hepatitis C related cirrhosis was the underlying etiology in most cases (93.9\%). Half the patients (53\%) were CTPA and $37.9 \%$ of patients were CTPB. Four hundred and fifty-four patients (59\%) had a single tumor. Portal vein thrombosis (PVT) was found in 165 patients $(21.4 \%)$ and 80 patients had extrahepatic spread.

Thirty-three patients presented in BCLC stage $0 ; 259$ patients (33.6\%) in stage A; $143(18.6 \%)$ in stage B; 265 $(34.4 \%)$ in stage C and $70(9.1 \%)$ stage D. All patients were referred to treatment according to the BCLC stage and AASLD recommended therapies (Bruix and Sherman, 2011).

In very early and early stages, $192(65.8 \%)$ received resection, ablation, liver transplantation or TACE for unresectable single tumors (more than $5 \mathrm{~cm}$ in diameter). Thirty-two (11\%) received downward treatment stage migration recommended for stage B (TACE, because they were not suitable for resection and their lesion was considered difficult for local ablation under ultrasound guidance) and 54 patients $(18.5 \%)$ did not receive active treatment (Table 1$)$. Only 14 patients $(4.8 \%)$ received a living donor liver transplant. Eleven and three patients received TACE and sorafenib respectively as second line treatment after failure of local ablation. Reasons for divergence from AASLD recommendations are shown in table 2. Patients' refusal or elevated portal pressure was the main reason for the low resection rate. Technical reasons (difficult tumor site) were the main reason for treatment stage migration in this stage (Table 2).

The median survival of patients in whom treatment stage was migrated downward was lower than patients who received the recommended treatment (15 months vs 36 months, $\mathrm{p}<0.001$ ) (Table 3, Figure 1A).

Of the 292 patients in this group, $216(71.8 \%)$ were within Milan criteria and were candidates for liver transplantation. The main reasons for the low transplant rate were the absence of a suitable donor (living donor transplants $\{$ LDLT $\}$ only are performed in Egypt, and availability of a suitable related donor limits transplant for most patients), a high AFP level $>1,000 \mathrm{ng} / \mathrm{ml}$, or limited resources (LTx is only partially reimbursed, and patients have to pay a minimum of $\$ 25,000$ out of pocket).

Table 3. Survival of Patients within Each Barcelona Clinic Liver Cancer Stage According to Real Life Treatment

\begin{tabular}{|c|c|c|c|c|}
\hline BCLC & Therapy & No. of patients & Median $(95 \% \mathrm{CI})$ & $\mathrm{p}$ \\
\hline \multirow{2}{*}{ A } & Recommended & 192 & $36.1(31.7-41.6)$ & \multirow{2}{*}{$<0.001$} \\
\hline & Downward treatment stage migration & 100 & $15(10.1-20.3)$ & \\
\hline \multirow{3}{*}{ B } & Recommended & 89 & $17.2(13-21.3)$ & 0.053 \\
\hline & Downward treatment stage migration & 49 & $12.2(7.1-16.7)$ & $* \mathrm{P} 1=0.07$ \\
\hline & Upward treatment stage migration & 5 & $15.2(9.1-21.4)$ & $* * \mathrm{P} 2=0.21$ \\
\hline \multirow{3}{*}{$\mathrm{C}$} & Recommended & 23 & $9.1(7.5-10.7)$ & 0.46 \\
\hline & Downward treatment stage migration & 214 & $8.1(6.3-9.9)$ & $* \mathrm{P} 1=0.97$ \\
\hline & Upward treatment stage migration & 28 & $11.2(8.2-14.2)$ & $* * \mathrm{P} 2=0.34$ \\
\hline \multirow{2}{*}{$\mathrm{D}$} & Recommended & 69 & $14.2(11.4-17)$ & \multirow{2}{*}{0.34} \\
\hline & Upward treatment stage migration & 1 & 16 & \\
\hline
\end{tabular}

*P1: comparison between survival time in patients who received recommended treatment and those who received downward treatment migration $* * \mathrm{P} 2$ : comparison between survival time in patients who received recommended treatment and those who received upward treatment migration 
Alzhraa Alkhatib et al

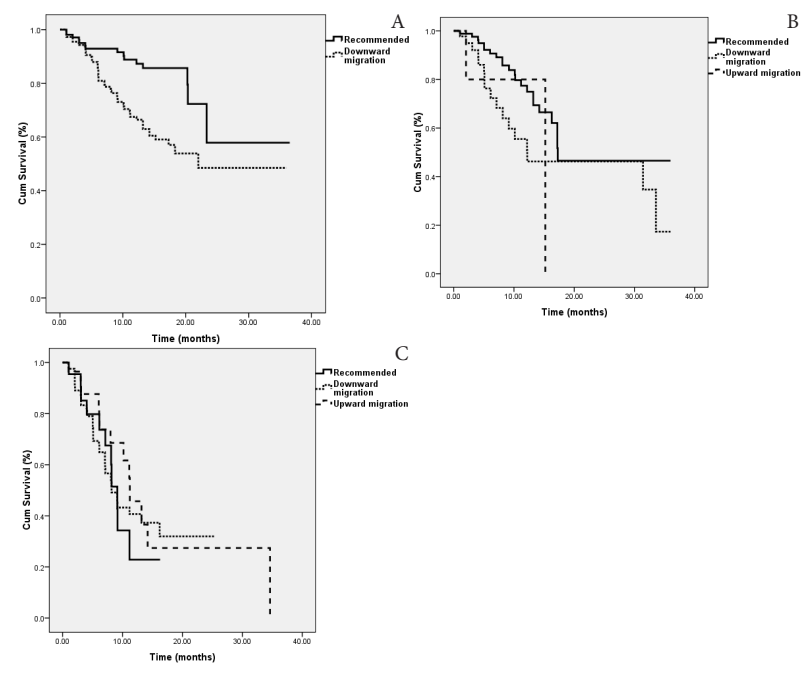

Figure 1. A) Survival curve for stage A) patients according to received treatment: recommended upward or downward treatment stage migration. B) Survival curve for stage B) patients according to received treatment; recommended, upward or downward treatment stage migration. C) Survival curve for stage $C$ ) patients according to received treatment; recommended upward or downward treatment stage migration

Among BCLC stage B patients, 86 of 143 (60.1\%) received TACE, including three who received combined RFA and TACE, and three patients received LDLT (2.1\%). Treatment stage was migrated upwards in 5 patients (3.5\%). For 49 patients $(34.3 \%)$, the treatment stage was migrated downward: 15 patients received sorafenib and 34 patients received supportive care (Table 1). The deviations were mainly due to large tumor size and diffuse liver involvement, impaired liver function (CTP score B9) or patients' refusal (Table 2). There was no improvement in median survival of patients for whom treatment stage was migrated upwards (15.2 months) compared to those who received recommended treatment (17.2 months). Median survival was slightly shorter in those for whom treatment stage was migrated downwards (12.2 months) (Table 3, Figure 1B).

BCLC stage $\mathrm{C}$ included 265 patients. Only 23 patients (7.5\%) received the recommended therapy: 20 received sorafenib $(7.5 \%)$ and three patients were within Milan and received LTx $(1.1 \%)$. Thirty-one patients received upward treatment stage migration: 22 received TACE $(8.3 \%)$, three received RFA (1.1\%), one received combined RFA and TACE, and two patients underwent hepatic resection $(0.8 \%)$. Most patients $(80.8 \%)$ received downward treatment stage migration; with 210 patients receiving supportive care $(79.2 \%)$, three patients received systemic chemotherapy, and one patient received radiotherapy (Table 1).

There was no difference in overall survival between patients for whom treatment stage was migrated downwards and those who received recommended treatment, with slight increase in survival in those for whom treatment stage was migrated upwards (11.2 vs. 8.1 months, $\mathrm{p}=0.4$ ) (Table 3, Figure 1C). Patients' treatment was deviated in this stage mainly due to lack of reimbursement for sorafenib owing to its high cost, or due to impaired liver function.

In stage $\mathrm{D}, 63$ patients were treated by supportive care and one patient received TACE. Ten patients were within Milan criteria and were offered LTx and six of them underwent LDLT.

Of 770 patients included in our study, 373(48.4\%) patients were treated according to BCLC recommendations, and $397(51.6 \%)$ were deviated in the form of upward treatment stage migration in 34 patients $(4.5 \%)$ and downward treatment stage migration in $363(47.1 \%)$. Deviations were $34.2 \%$ in stage 0 and A, $37.7 \%$ in stage B and $92.4 \%$ in stage $\mathrm{C}$.

\section{Discussion}

In the past ten years, 17 guidelines for management of HCC have been published worldwide (Song et al., 2012). However, adherence to guidelines is seldom as optimal as expected, and adherence is critical in translating recommendations into improved outcomes (Borzio and Sacco, 2013). Few studies evaluated the implementation of guidelines' algorithms for therapy of HCC (D'Avola et al., 2011; Kim et al., 2011; Radu et al., 2013), but none has done so in resource-limited settings, in which the incidence of HCC is reported to be remarkably increasing (Jada et al., 2014).

In this study we analyzed frequency of adherence to recommended therapy and the effect of treatment deviation from guideline recommendation on survival in each BCLC stage in a public tertiary referral hospital for liver disease in Egypt. Only $48.4 \%$ of the patients received the recommended treatment, $4.5 \%$ of patients received upward treatment stage migration and $47.1 \%$ received downward treatment stage migration. The major discrepancy occurred in patients with BCLC stage C. The deviation from treatment was mainly due to the lack of reimbursement for sorafenib, in addition to patients' preference in some cases.

Of patients in stage 0 and A, only $65.8 \%$ received curative treatment, a comparable rate to that reported by D'Avola (66\%) (D'Avola et al., 2011). This is a higher rate than that reported by another Egyptian center (Abdelaziz et al., 2014). Survival in the present study was affected more (36 months vs. 117 months) than in D'Avola's. This is probably due to the low transplant rate in our series $(4.8 \%$ vs. $34 \%$ ) and the shorter period of follow up in our study.

Three percent of the patients included in BCLC B received upward treatment stage migration; a percentage that is very small compared to other studies (D'Avola et al., 2011; Radu et al., 2013), while $62.2 \%$ were treated by TACE. Survival of patients treated according to BCLC (TACE) was better than those who received treatment stage migration.

Although sorafenib has proven survival benefits in BCLC C and is recommended by several guidelines, only $7.5 \%$ of our patients with BCLC stage C received sorafenib. Sorafenib is expensive, with an average cost of US $\$ 6,000$ per month in different countries (Cabrera and Nelson, 2010), and can only be applied in countries 
with extensive financial resources for healthcare services (Parikh et al., 2008; Benson et al., 2009; Bruix and Sherman, 2011; Song et al., 2012). The monthly cost of sorafenib in Egypt is lower ( $\$ 3,000$ for a month's supply), yet this is close to the annual per-capita income, and is not reimbursed by national or most private insurance, and most patients have to pay out of their own resources.

In this study, treatment stage migration did not significantly affect the survival in patients with BCLC stages $\mathrm{B}$ or $\mathrm{C}$ in contrast to BCLC stages A which was significantly lower in patients who did not receive recommended therapy. Other studies found better survival for patients with upward treatment stage migration than patients treated according to the BCLC recommendations (Ruzzenente et al., 2009; Pinter et al., 2012; Radu et al., 2013). Very few patients in this study underwent LDLT. The lack of suitable living-related liver donors and the lack of reimbursement are important reasons why LTx was not performed when indicated.

Few studies reported the adherence rate to the recommended therapy which ranged from $40 \%$ to $62 \%$ in resource-rich settings (D'Avola et al., 2011; Kim et al., 2011; Radu et al., 2013). Upward treatment stage migration has been found in $11 \%-21 \%$, and downward treatment stage migration in $17 \%-30 \%$, with most deviation $(90 \%)$ being in stage B. The main reason for not following AASLD recommendations in these studies were the low accessibility to TACE and transplantation, the long waiting time for sorafenib therapy and its expected low survival benefit beside its adverse effects.

In a study by Borzio and his colleagues the most relevant deviation from the AASLD guidelines was an overall downward treatment stage migration (Borzio et al., 2013). The authors drew attention to the most relevant barrier contributing to the deviations in their population, which were the presence of a large number of elderly patients with relevant extrahepatic co-morbidities.

The main limitation of our study was the low accessibility to LT and sorafenib as main treatment options in our center. This study shows that limited resources were the cause for deviation from guideline recommendation in most cases. The lack of reimbursement for sorafenib prevented more than $90 \%$ of patients from accessing recommended therapy. Although $28 \%$ of the patients were within Milan criteria and would have been candidates for transplantation, access to liver transplantation was limited, mainly by the lack of living related donors. However, for those patients who had a willing suitable donor, partial reimbursement and the need to pay $75 \%$ of the cost was prohibitive for many. This impacted patient survival, and should alert policy-makers and healthcare planners to allocate more resources to these groups of patients, and to strive to make recommended therapy affordable to patients in need.

Our results are probably representative of other resource-limited settings, where treatments are available but not within patients' affordability. These results, however, cannot be generalized to resource-rich countries where treatments are available and completely reimbursed for all patients.

\section{References}

Abdelaziz AO, Elbaz TM, Shousha HI, et al (2014). Survival and prognostic factors for hepatocellular carcinoma: an egyptian multidisciplinary clinic experience. Asian Pac J Cancer Prev, 15, 3915-20.

Benson AB $3^{\text {rd }}$, Abrams TA, Ben-Josef E, et al (2009). NCCN clinical practice guidelines in oncology: hepatobiliary cancers. J Natl Compr Canc Netw, 7, 350-91.

Borzio M, Fornari F, De Sio I, et al (2013). Adherence to AASLD guidelines for the management of hepatocellular carcinoma: results of an Italian field-practice multicenter study. Future Oncol, 9, 283-94.

Borzio M, Sacco R (2013). Nonadherence to guidelines in the management of hepatocellular carcinoma: an Italian or universal phenomenon? Future Oncol, 9, 465-7.

Bruix J, Sherman M, Llovet JM, et al (2001). Clinical management of hepatocellular carcinoma. Conclusions of the Barcelona- 2000 EASL conference. J Hepatol, 35, 421-30.

Bruix J, Sherman M (2005). AASLD practice guidelines. management of hepatocellular carcinoma. Hepatol, 42, 1208-36.

Bruix J, Sherman M (2011). Management of hepatocellular carcinoma: an update. american association for the study of liver diseases (AASLD) practice guideline. Hepatol, 53, 1020-35.

Cabrera R, Nelson DR (2010). Review article: the management of hepatocellular carcinoma. Aliment Pharmacol Ther, 31, 461-76.

D'Avola D, Inarrairaegui M, Pardo F, et al (2011). Prognosis of hepatocellular carcinoma in relation to treatment across BCLC stages. Ann Surg Oncol, 18, 1964-71.

De Lope CR, Tremosini S, Forner A, Reig M, Bruix J (2012). Management of HCC. J Hepatol, 56, 75-87.

European Association For The Study Of The Liver (2012). European organisation for research and treatment of cancer. EASL-EORTC clinical practice guidelines: management of hepatocellular carcinoma. J Hepatol, 56, 908-43.

Forner A, Reig ME, de Lope CR, Bruix J (2010). Current strategy for staging and treatment: the BCLC update and future prospects. Semin Liver Dis, 30,61-74.

Jaka H, Mshana SE, Rambau PF, et al (2014). Hepatocellular carcinoma: clinicopathological profile and challenges of management in a resource-limited setting. World J Surg Oncol, 2014, 12, 246.

Kaplan E, Meier P (1958). Nonparametric estimation from incomplete observations. J Am Stat Assoc, 53, 457-81.

Kim SE, Lee HC, Kim KM, et al (2011). Applicability of the BCLC staging system to patients with hepatocellular carcinoma in Korea: analysis at a single center with a liver transplant center. Korean J Hepatol, 17, 113-9.

Llovet JM, Ricci S, Gane E, et al (2008). Sorafenib in advanced hepatocellular carcinoma. $N$ Engl J Med, 359, 378-90.

Oken MM, Creech RH, Tormey DC, et al (1982). Toxicity and response criteria of the eastern cooperative oncology group. Am J Clin Oncol, 5, 649-55.

Omata M, Lesmana LA, Tateishi R, et al (2010). Asian pacific association for the study of the liver consensus recommendations on hepatocellular carcinoma. Hepatol Int, 4, 439-74.

Parikh P, Malhotra H, Jelic S (2008). ESMO guidelines working group. hepatocellular carcinoma: ESMO clinical recommendations for diagnosis, treatment and follow-up. Ann Oncol, 19, 27-8.

Pinter M, Hucke F, Graziadei I, et al (2012). Advanced-stage hepatocellular carcinoma: transarterial chemoembolization versus sorafenib. Radiol, 263, 590-9. 
Alzhraa Alkhatib et al

Radu P, Ioana G, Iancu C, et al (2013). Treatment of hepatocellular carcinoma in a tertiary romanian center. deviations from BCLC recommendations and influence on survival rate. J Gastrointestin Liver Dis, 22, 291-7.

Ruzzenente A, Capra F, Pachera S, et al (2009). Is liver resection justified in advanced hepatocellular carcinoma? results of an observational study in 464 patients. J Gastrointest Surg, 13, 1313-20.

Ryder SD, British Society of Gastroenterology (2003). Guidelines for the diagnosis and treatment of hepatocellular carcinoma (HCC) in adults. Gut, 52, iii1-8. 10.1136/gut.52. suppl_3.iii1

Song P, Tobe RG, Inagaki Y, et al (2012). The management of hepatocellular carcinoma around the world: a comparison of guidelines from 2001 to 2011. Liver Int, 32, 1053-63.

Su Cheng-Hao, Lin Y , Cai L (2013). Genetic factors, viral infection, other factors and liver cancer: an update on current progress. Asian Pac J Cancer Prev, 14, 4953-60. 